\title{
Inflammatory response of esophageal epithelium in combined-type esophagitis in rats: A transcriptome analysis
}

\author{
YUJI NAITO $^{1}$, MASAAKI KURODA ${ }^{2}$, KAZUHIKO UCHIYAMA ${ }^{2}$, KATSURA MIZUSHIMA ${ }^{2}$, \\ SATOMI AKAGIRI ${ }^{2}$, TOMOHISA TAKAGI ${ }^{3}$, OSAMU HANDA ${ }^{3}$, SATOSHI KOKURA ${ }^{3}$, \\ NORIMASA YOSHIDA ${ }^{4}$, HIROSHI ICHIKAWA ${ }^{5}$ and TOSHIKAZU YOSHIKAWA Y, $^{1,2,3}$ \\ ${ }^{1}$ Department of Medical Proteomics, ${ }^{2}$ Inflammation and Immunology, ${ }^{3}$ Department of Biomedical Safety Science, \\ and ${ }^{4}$ Molecular Gastroenterology and Hepatology, Graduate School of Medical Science, Kyoto Prefectural \\ University of Medicine, Kyoto 602-8566; ${ }^{5}$ Department of Food Sciences and Nutritional Health, \\ The Faculty of Human Environment, Kyoto Prefectural University, Kyoto 606-8522, Japan
}

Received May 15, 2006; Accepted July 17, 2006

\begin{abstract}
Recent studies have shown that esophageal mucosal inflammatory response is involved in the pathophysiology of gastro-esophageal reflux disease. The aim of the present study was to identify specific gene expression profiles of the esophageal mucosa in a rat model of combined-type chronic reflux esophagitis. Esophagogastroduodenal anastomosis was carried out in male Wistar rats by anastomosing the jejunum to the gastroesophageal junction under diethyl-ether inhalation anesthesia. Esophageal epithelial cells were obtained from esophagi of rats by laser capture microdissection. Preparation of cRNA and target hybridization were performed according to the Affymetrix GeneChip eukaryotic small sample target labeling assay protocol. The gene expression profile was evaluated by the rat toxicology U34 GeneChip. Array data analysis was carried out using Affymetrix GeneChip operating software, Ingenuity pathway analysis software, and Gene Springs software. A comparison between esophagitis and sham-operated rats 2 weeks after the operation revealed that 368 probes $(36 \%)$ were significantly affected, i.e. 185 probes were up-regulated, and 183 probes were down-regulated, both at levels of at least 1.5 -fold in the esophagitis rats. Ingenuity signal analysis of 207 affected probes revealed the interleukin-6 signaling pathway as the most significantly affected caronical pathway. In addition, the expression of many genes associated with cytokine and transcription factor was enhanced in the esophagitis rats. This transcriptome approach provided insight into genes and putative genetic pathways thought to be affected by stimulation with gastroduodenal refluxates.
\end{abstract}

Correspondence to: Dr Yuji Naito, Department of Medical Proteomics, Kyoto Prefectural University of Medicine, KawaramachiHirokoji, Kamigyo-ku, Kyoto 602-8566, Japan

E-mail: ynaito@koto.kpu-m.ac.jp

Key words: esophagus, GeneChip, inflammation, reflux esophagitis, transcriptome

\section{Introduction}

The pathophysiology of gastro-esophageal reflux disease (GERD) involves contact of the esophageal epithelium with gastric/duodenal juice in the refluxate. Several studies have shown that esophageal mucosal immune and inflammatory responses, characterized by specific cytokine and chemokine profiles, may determine the diversity of esophageal phenotypes of GERD. Fitzgerald et al (1) first reported that reflux esophagitis is characterized by an acute inflammatory response with significantly increased levels of the proinflammatory cytokines [interleukin-1ß (IL-1ß), IL- 8 , and interferon- $\gamma$ ] compared with noninflamed squamous esophagus. Isomoto et al (2) have also demonstrated that the presence of intraepithelial neutrophils and eosinophils, which also indicate reflux esophagitis, is associated with high levels of IL-8 and regulated on activation normal T-cell expressed and presumably secreted (RANTES), respectively, and that the IL-8 levels are significantly decreased after proton pump inhibitor treatment. We have also investigated the relationship between the IL-8/monocyte chemoattractant protein 1 (MCP-1) mRNA expression and endoscopic grading of reflux esophagitis according to the Los Angeles classification (3). The expression of IL-8 mRNA determined by real-time PCR correlates with the endoscopic severity of GERD, and increases in patients with nonerosive reflux disease (NERD) compared to normal subjects. There is no correlation between MCP-1 mRNA expression and endoscopic severity, or between the severity of subjective symptoms (QUEST score) and endoscopic grading. These data indicate that chemokine production locally in the esophageal mucosa may be involved in the development and progression of reflux esophagitis, and that gastric acid may play a role in the induction of esophageal inflammation.

Immunohistochemical staining has provided evidence that esophageal epithelial cells produce IL-8 protein $(1,3)$. In order to confirm the epithelial cells as a potential source of cytokines and to identify the molecular mechanism involved in IL-8 production, we have determined whether cultured human esophageal epithelial cells (HEEC) produce IL-8 (4). Stimulation of HEEC with cholic acid or taurochenodeoxycholic 
acid results in IL- 8 production via p38 mitogen-activated protein kinase (MAPK) phosphorylation-dependent pathway. However, the precise mechanism of inflammatory response in esophageal epithelium, especially in vivo, has not been fully determined. Some studies of reflux esophagitis have been based on analysis of the expression of a single molecule, or of a relatively limited number of these molecules in esophageal mucosa. DNA microarray techniques have become available that have enabled simultaneous characterization of the mRNA expression pattern of a large number of genes. In the present study, we identified specific gene expression profiles of esophageal epithelial cells in experimental esophagitis, which was recently created by esophagogastroduodenal anastomosis in rats as a model of combined-type chronic reflux esophagitis (5).

\section{Materials and methods}

Reagents. All chemicals were prepared immediately before use. An RNeasy Mini kit was purchased from Qiagen (Valencia, CA) and Rat Toxicology GeneChip U34 array and Eukaryotic Small Sample Target Labeling Assay kit were from Affymetrix (Santa Clara, CA). All other chemicals used were of reagent grade.

Rat model of gastroduodenal reflux. Seven-week-old male Wistar rats (Nihon SLC, Hamamatsu, Japan) weighing 200$250 \mathrm{~g}$ were used. The rats were housed two per cage under standard laboratory conditions (room temperature, $22 \pm 2^{\circ} \mathrm{C}$; relative humidity, 55 $\pm 5 \%$; and a 12-h light/dark cycle). All procedures were prospectively approved by the animal care and use committee of Kyoto Prefectural University of Medicine. Animals were acclimatized in the facility for 1 week before the experiment. Prior to surgery, animals were fasted for $24 \mathrm{~h}$. General anesthesia was induced by diethyl-ether inhalation. A side-to-side esophagogastroduodenostomy was created to induce mixed gastroduodenal reflux according to the previous report (5). At first the transitional region between the forestomach and the glandular portion was ligated with a 1-0 silk thread. A longitudinal incision of approximately $7 \mathrm{~mm}$ in length was then made on the antimesenteric border of the duodenum. A second incision was made longitudinally along the lower esophagus anteriorly extending $7 \mathrm{~mm}$ distally into the proximal stomach. A side-to-side anastomosis was fashioned between the two openings using 7 polypropylene 7-0 sutures. The abdominal wall was closed in two layers with 1-0 silk sutures. Animals were fed only water for $48 \mathrm{~h}$ after surgery.

Laser capture microdissection, cRNA amplification, and GeneChip hybridization. We used laser-assisted microdissection to obtain cell-specific RNA. Esophageal epithelial cells were identified on cryostat sections $(8 \mu \mathrm{m})$ of specimens obtained from the esophagus of the rat, and the cells were isolated by laser-assisted microdissection using an LM200 system (Olympus, Tokyo, Japan). A sample containing 200 cells was collected from each esophagus. Although laser capture microdissection can be used to produce cell-specific RNA, this method is limited by the amount of RNA that can be realistically obtained from captured populations of cells, making it likely that the yield will be insufficient for the commonly used GeneChip assay. To overcome this obstacle, our experiments were performed according to the Affymetrix GeneChip eukaryotic small sample target labeling assay protocol (version II). According to our protocol $(6,7)$, we succeeded in obtaining a sufficient amount of biotinylated cRNA to perform GeneChip analysis from the small amount of esophageal epithelial cells obtained by laser-captured microdissection.

The total RNA was extracted using a Qiagen RNeasy kit and treated with DNase to remove any residual genomic DNA. Briefly, for the first-strand cDNA synthesis, the total RNA sample $(1 \mu 1)$, mixed with T7-Oligo(dT) promoter primer $(5 \mu \mathrm{M}, 1 \mu \mathrm{l})$, was incubated at $70^{\circ} \mathrm{C}$ in a thermal cycler for $6 \mathrm{~min}$, cooled to $4^{\circ} \mathrm{C}$ for $2 \mathrm{~min}$, and then reverse-transcribed for $1 \mathrm{~h}$ at $42^{\circ} \mathrm{C}$ with $3 \mu \mathrm{l}$ of the RT Premix 1 . The sample was then heated at $70^{\circ} \mathrm{C}$ for $10 \mathrm{~min}$ to inactivate the SuperScript $\mathrm{II}$, and was cooled to $4^{\circ} \mathrm{C}$. Second-strand cDNA synthesis was carried out by adding $32.5 \mu \mathrm{l}$ of SS Premix 1 and incubating the sample for $2 \mathrm{~h}$ at $16^{\circ} \mathrm{C}$. The resulting cDNA was treated with $1 \mu 1 \mathrm{~T} 4 \mathrm{DNA}$ polymerase $(5 \mathrm{U} / \mu \mathrm{l})$ for $10 \mathrm{~min}$ at $16^{\circ} \mathrm{C}$, and was cleaned by ethanol precipitation. After the in vitro transcription, the first-cycle cRNA was cleaned using the RNeasy Mini protocol for RNA cleanup from the handbook accompanying the RNeasy Mini kit for cRNA purification (Qiagen). For the second cycle of amplification and labeling, the cRNA sample was mixed with random primers $(0.2 \mu \mathrm{g} / \mu \mathrm{l})$, incubated at $70^{\circ} \mathrm{C}$ for $10 \mathrm{~min}$, cooled on ice for $2 \mathrm{~min}$, and incubated at $42^{\circ} \mathrm{C}$ for $1 \mathrm{~h}$ with $5 \mu \mathrm{l}$ of the RT Premix 2 . Second-strand cDNA synthesis was carried out by mixing the sample with $5 \mu \mathrm{M}$ T7-Oligo(dT) promoter primer, incubating the resulting mixture at $70^{\circ} \mathrm{C}$ for $6 \mathrm{~min}$, cooling it at $4^{\circ} \mathrm{C}$, and re-incubating the sample with $62 \mu 1$ of SS Premix 2. The resulting cDNA was treated with $1 \mu \mathrm{l}$ T4 DNA polymerase $(5 \mathrm{U} / \mu \mathrm{l})$ for $10 \mathrm{~min}$ at $16^{\circ} \mathrm{C}$, and cleaned up by ethanol precipitation. To perform in vitro transcription and labeling with the ENZO BioArray high yield RNA transcript labeling kit, the dried double-stranded cDNA pellet was incubated at $37^{\circ} \mathrm{C}$ for $4 \mathrm{~h}$ with $40 \mu \mathrm{l}$ of the following reagents: $22 \mu \mathrm{l}$ DEPC-treated water, $4 \mu 1$ 10X HY reaction buffer, $4 \mu 1$ 10X biotin labeled ribonucleotides, $4 \mu 1$ 10X DTT, $4 \mu 1$ 10X RNase inhibition mix, and $2 \mu 120 \mathrm{X}$ T7 RNA polymerase. Labeled cRNA target was cleaned up using RNeasy columns. The fragmentation, hybridization, washing, and staining were carried out according to the instructions described in the GeneChip expression analysis technical manual. Affymetrix GeneChip arrays were hybridized with the biotinylated products ( $5 \mu \mathrm{g} / \mathrm{chip}$ ) for $16 \mathrm{~h}$ at $45^{\circ} \mathrm{C}$ using the manufacturer's hybridization buffer. After washing the arrays, hybridized RNA was detected by staining the sample with streptavidin-phycoerythrin. The DNA chips were scanned using a specially designed confocal scanner (GeneChip Scanner 3000, Affymetrix).

Data analysis. Array data analysis was carried out using Affymetrix GeneChip operating software (GCOS) version 1.0. GCOS analyzes image data and computes an intensity value for each probe cell. Briefly, mismatch probes act as specificity controls that allow the direct subtraction of both background and cross-hybridization signals. To determine the quantitative RNA abundance, the average difference values (i.e., gene expression levels) representing the perfect match- 
Table I. Genes up-regulated (determined as I by GCOS) at least 1.5-fold in esophageal mucosa 2 weeks after esophagogastrojejunostomy (EGJ-stomy).

\begin{tabular}{|c|c|c|c|c|c|}
\hline \multirow[b]{2}{*}{ Accession no. } & \multirow[b]{2}{*}{ Gene title } & \multicolumn{2}{|c|}{ Average difference ${ }^{a}$} & \multicolumn{2}{|c|}{ Fold change $^{\mathrm{b}}$} \\
\hline & & Sham & EGJ-stomy & Log ratio & Ratio \\
\hline AF048687_s_at & UDP-Gal:ßGlcNAc ß 1,4-galactosyltransferase, polypeptide 6 & 23.3 & 301.6 & 3.4 & 10.56 \\
\hline rc_AA874919_at & mismatch repair protein & 17.6 & 106.6 & 3.0 & 8.00 \\
\hline rc AI045249_at & $\begin{array}{l}\text { Transcribed sequence with moderate similarity to protein ref: } \\
\text { NP_149107.1 (H. sapiens) }\end{array}$ & 24.9 & 181.8 & 2.8 & 6.96 \\
\hline rc_AI229655_at & Transcribed sequences & 96.1 & 432.3 & 2.3 & 4.92 \\
\hline X60767mRNA_s_at & cell division cycle 2 homolog A (S. pombe) & 27.2 & 136.0 & 2.3 & 4.92 \\
\hline AF025670_g_at & caspase 6 & 67.8 & 140.5 & 2.0 & 4.00 \\
\hline U39207_at & cytochrome P450 4F5 & 50.6 & 162.6 & 1.9 & 3.73 \\
\hline rc_AA875594_s_at & FK506-binding protein $1 \mathrm{a}$ & 44.6 & 135.1 & 1.8 & 3.48 \\
\hline X96394_at & ATP-binding cassette, sub-family C (CFTR/MRP), member 1 & 50.7 & 149.6 & 1.7 & 3.25 \\
\hline rc_AA926129_at & $\begin{array}{l}\text { Transcribed sequence with strong similarity to protein ref: } \\
\text { NP_446139.1 (R. norvegicus) }\end{array}$ & 39.5 & 145.9 & 1.6 & 3.03 \\
\hline K01721mRNA_s_at & cytochrome P450,2b19 & 46.5 & 178.2 & 1.5 & 2.83 \\
\hline D85035_g_at & dihydropyrimidine dehydrogenase & 92.5 & 258.9 & 1.4 & 2.64 \\
\hline AF003523_s_at & bcl-2 associated death agonist & 39.2 & 87.3 & 1.3 & 2.46 \\
\hline U53922_at & DnaJ-like protein & 376.4 & 1068.2 & 1.2 & 2.30 \\
\hline AFFX_Rat_beta-actin_3_st & & 106.8 & 246.3 & 1.1 & 2.14 \\
\hline rc_AI012589_s_at & glutathione-S-transferase, pi 1 & 356.9 & 850.2 & 1.1 & 2.14 \\
\hline X13933_s_at & Calmodulin 1 (phosphorylase kinase, $\delta$ ) & 236.8 & 497.8 & 1.1 & 2.14 \\
\hline J04791_s_at & ornithine decarboxylase 1 & 14.2 & 50.9 & 1.0 & 2.00 \\
\hline L26267_at & nuclear factor $\kappa$ B p105 subunit & 218.2 & 466.7 & 1.0 & 2.00 \\
\hline M14050_s_at & heat shock $70 \mathrm{k} 0$ protein 5 & 390.9 & 837.3 & 1.0 & 2.00 \\
\hline M61875_s_at & CD44 antigen & 243.3 & 559.7 & 1.0 & 2.00 \\
\hline rc_AA799889_at & Transcribed sequences & 103.6 & 197.5 & 1.0 & 2.00 \\
\hline D84450_at & ATPase, $\mathrm{Na}^{+} / \mathrm{K}^{+}$transporting, $B 3$ polypeptide & 921.0 & 2215.3 & 0.9 & 1.87 \\
\hline rc_AA818226_s_at & cytochrome $\mathrm{c}$ oxidase, subunit $4 \mathrm{a}$ & 2699.6 & 5211.6 & 0.9 & 1.87 \\
\hline rc_AI231354_g_at & stress activated protein kinase $\alpha$ II & 154.2 & 313.3 & 0.9 & 1.87 \\
\hline Z78279_g_at & collagen, type $1, \alpha 1$ & 597.9 & 1050.2 & 0.9 & 1.87 \\
\hline M28255_s_at & cytochrome c oxidase, subunit Villa & 1900.6 & 3482.9 & 0.8 & 1.74 \\
\hline rc_AI177256_at & Transcribed sequences & 134.1 & 166.9 & 0.8 & 1.74 \\
\hline U49930_at & caspase 3 & 47.8 & 73.1 & 0.8 & 1.74 \\
\hline J02810mRNA_s_at & glutathione S-transferase, mu 1 & 2511.9 & 3808.3 & 0.7 & 1.62 \\
\hline J05132_s_at & UDP glycosyltransferase 1 family, polypeptide A6 & 2670.0 & 4402.5 & 0.7 & 1.62 \\
\hline rc_AA955983_at & $\begin{array}{l}\text { Transcribed sequence with weak similarity to protein sp: } \\
\text { P20291 (R. norvegicus) }\end{array}$ & 726.5 & 1176.8 & 0.7 & 1.62 \\
\hline rc_AI176170_at & FK506-binding protein $1 \mathrm{a}$ & 594.9 & 841.5 & 0.7 & 1.62 \\
\hline U47315_s_at & brain protein 44 -like & 816.6 & 1563.4 & 0.7 & 1.62 \\
\hline U47316_s_at & $\begin{array}{l}\text { Transcribed sequence with strong similarity to protein ref: } \\
\text { NP_038746.1 (M. musculus) }\end{array}$ & 327.4 & 471.5 & 0.7 & 1.62 \\
\hline X54793_at & heat shock protein 60 (liver) & 1422.7 & 2552.6 & 0.7 & 1.62 \\
\hline X60328_at & cytosolic epoxide hydrolase & 13.2 & 63.9 & 0.7 & 1.62 \\
\hline D00569_g_at & 2,4-dienoyl CoA reductase 1 , mitochondrial & 88.4 & 127.0 & 0.6 & 1.52 \\
\hline D16554_at & polyubiquitin & 4334.8 & 6691.0 & 0.6 & 1.52 \\
\hline K00750exon\#2-3_at & & 142.2 & 219.4 & 0.6 & 1.52 \\
\hline K00750exon\#2-3_g_at & & 976.9 & 1866.2 & 0.6 & 1.52 \\
\hline M24604_at & Proliferating cell nuclear antigen & 721.4 & 997.2 & 0.6 & 1.52 \\
\hline M76767_s_at & fatty acid synthase & 214.4 & 346.8 & 0.6 & 1.52 \\
\hline rc_AA850781_at & $\begin{array}{l}\text { Transcribed sequence with strong similarity to protein ref: } \\
\text { NP_080628.1 (M. musculus) }\end{array}$ & 45.3 & 65.9 & 0.6 & 1.52 \\
\hline rc_AA875327_g_at & $\begin{array}{l}\text { Transcribed sequence with weak similarity to protein ref: } \\
\text { NP_072143.1 (R. norvegicus) }\end{array}$ & 425.5 & 564.4 & 0.6 & 1.52 \\
\hline rc_AA893185_at & $\begin{array}{l}\text { Transcribed sequence with moderate similarity to protein ref: } \\
\text { NP_002483.1 (H. sapiens) }\end{array}$ & 364.7 & 556.2 & 0.6 & 1.52 \\
\hline rc_AA899854_at & topoisomerase (DNA) $2 \alpha$ & 392.4 & 592.1 & 0.6 & 1.52 \\
\hline rc_AI007614_at & $\begin{array}{l}\text { Transcribed sequence with strong similarity to protein ref: } \\
\text { NP_032722.1 (M. musculus) }\end{array}$ & 352.4 & 517.5 & 0.6 & 1.52 \\
\hline rc_AI104520_s_at & cytochrome c oxidase, subunit VIa, polypeptide 1 & 951.6 & 1738.6 & 0.6 & 1.52 \\
\hline rc_AI171355_s_at & & 1160.1 & 1907.7 & 0.6 & 1.52 \\
\hline U77933_at & caspase 2 & 367.4 & 623.8 & 0.6 & 1.52 \\
\hline
\end{tabular}

a Average difference indicates the level of expression of the gene. ${ }^{b}$ Fold changes in average difference values were calculated using an Affymetrix software algorithm (GCOS ver. 1.0). 
Table II. Genes down-regulated (determined as D by GCOS) at least 1.5-fold in esophageal mucosa 2 weeks after esophagogastrojejunostomy (EGJ-stomy).

\begin{tabular}{|c|c|c|c|c|c|}
\hline \multirow[b]{2}{*}{ Accession no. } & \multirow[b]{2}{*}{ Gene title } & \multicolumn{2}{|c|}{ Average difference ${ }^{a}$} & \multicolumn{2}{|c|}{ Fold change $^{\mathrm{b}}$} \\
\hline & & Sham & EGJ-stomy & Log ratio & Ratio \\
\hline J02612mRNA_s_at & UDP glycosyltransferase 1 family, polypeptide A6 & 672.9 & 293.2 & -0.6 & 0.66 \\
\hline M55534mRNA_s_at & crystallin, $\alpha \mathrm{B}$ & 979.2 & 538.0 & -0.6 & 0.66 \\
\hline rc_AI179916_at & $\begin{array}{l}\text { Transcribed sequence with strong similarity to protein ref: } \\
\text { NP_057180.1 (H. sapiens) }\end{array}$ & 216.2 & 125.9 & -0.6 & 0.66 \\
\hline AFFX-BioB-5_at & & 552.5 & 310.4 & -0.7 & 0.62 \\
\hline U15211_at & Retinoic acid receptor, $\alpha$ & 3451.8 & 1991.1 & -0.7 & 0.62 \\
\hline $\mathrm{U} 17697 \mathrm{~s}$ at & cytochrome P450, subfamily 51 & 567.9 & 281.7 & -0.7 & 0.62 \\
\hline AF017393_at & cytochrome $\mathrm{P} 450$, subfamily $2 \mathrm{~F}$, polypeptide 1 & 3117.4 & 1902.1 & -0.8 & 0.57 \\
\hline AFFX-BioB-3_at & & 488.1 & 243.8 & -0.8 & 0.57 \\
\hline L17127_g_at & proteasome (prosome, macropain) subunit, $B$ type 4 & 692.2 & 378.3 & -0.8 & 0.57 \\
\hline AFFX-BioC-5_at & & 2013.3 & 1090.6 & -0.9 & 0.54 \\
\hline D87336_g_at & & 2395.0 & 1070.5 & -0.9 & 0.54 \\
\hline AFFX-BioDn-5_st & & 414.7 & 147.9 & -1.0 & 0.50 \\
\hline AFFX-BioDn-3_at & & 11897.4 & 5383.7 & -1.1 & 0.47 \\
\hline AFFX-CreX-3_at & & 25149.0 & 10238.9 & -1.1 & 0.47 \\
\hline AFFX-CreX-3_st & & 679.2 & 230.8 & -1.1 & 0.47 \\
\hline rc_AI169265_at & $\begin{array}{l}\text { Transcribed sequence with strong similarity to protein ref: } \\
\text { NP_076320.1 (M. musculus) }\end{array}$ & 1990.1 & 702.2 & -1.1 & 0.47 \\
\hline AFFX-BioC-3_at & & 1532.3 & 631.5 & -1.2 & 0.44 \\
\hline D87336_at & & 705.4 & 277.2 & -1.2 & 0.44 \\
\hline L32601_s_at & $20 \alpha$-hydroxysteroid dehydrogenase & 385.1 & 170.6 & -1.2 & 0.44 \\
\hline rc_AI231354_at & stress activated protein kinase $\alpha$ II & 137.9 & 46.1 & -1.2 & 0.44 \\
\hline AFFX-CreX-5_st & & 571.1 & 218.8 & -1.3 & 0.41 \\
\hline E03344cds_s_at & peroxisomal membrane protein 3 & 9.3 & 2.2 & -1.4 & 0.38 \\
\hline L03294_at & Lipoprotein lipase & 288.0 & 112.4 & -1.4 & 0.32 \\
\hline AFFX-BioDn-5_at & & 1501.0 & 546.9 & -1.5 & 0.35 \\
\hline AFFX-CreX-5_at & & 15387.0 & 5233.1 & -1.5 & 0.35 \\
\hline L03294_g_at & Lipoprotein lipase & 687.8 & 338.3 & -1.5 & 0.35 \\
\hline rc_AA945054_s_at & cytochrome b5 & 1326.9 & 178.2 & -2.5 & 0.18 \\
\hline X7̄9081mRNA_f_at & Cytochrome P450, subfamily IIC (mephenytoin 4-hydroxylase) & 553.5 & 42.6 & -2.7 & 0.15 \\
\hline J05156_s_at & cytochrome P450, subfamily $11 \mathrm{~A}$ & 117.1 & 15.0 & -2.8 & 0.14 \\
\hline J02585_at & stearoyl-Coenzyme A desaturase 1 & 205.7 & 17.2 & -3.4 & 0.09 \\
\hline rc_AI17̄8204_at & Transcribed sequences & 87.9 & 3.4 & -4.2 & 0.05 \\
\hline
\end{tabular}

${ }^{a}$ Average difference indicates the level of expression of the gene. ${ }^{b}$ Fold changes in average difference values were calculated using an Affymetrix software algorithm (GCOS ver. 1.0).

mismatch for each gene-specific probe family were calculated, and the fold changes in average difference values were determined according to Affymetrix algorithms and procedures as follows: I, increase; MI, marginal increase; D, decrease; MD, marginal decrease; and NC, no change. For the pathway analysis, gene probe set ID numbers were imported into the Ingenuity pathway analysis software (Ingenuity Systems, Mountain View, CA). The identified genes were mapped to genetic networks available in the Ingenuity database and were then ranked by a score. The score is the probability that a collection of genes is equal to or greater than the number in a network that could be achieved by chance alone. A score of 3 indicates that there is a $1 / 1000$ chance (significance $=0.001$ ) that the focus genes randomly occur in a network. Therefore, gene sets with scores of 3 or higher have a $99.9 \%$ confidence level of not being randomly generated. This score was used as the cut-off for identifying gene networks significantly affected by esophagitis. Using the signal intensity of genes selected using the following keywords: cytokine or transcriptional factor by a soft of NetAff $\mathrm{x}^{\mathrm{TM}}$ Analysis Center (Affymetrix), hierarchical-clustering analysis was performed with a GeneSpring software package, version 7.0 (Silicon Genetics, San Carlos, CA).

\section{Results and discussion}

In the present study, we used a high-density oligonucleotide microarray technique for the mRNA expression profile of esophageal epithelial cells in order to investigate the inflammatory reaction in a rat model of the combined-type chronic esophagitis. We used the GeneChip of rat toxicology U34 array (Affymetrix), which contained 1,031 probes selected from the UniGene database. Comparison of the expression profiles from sham-operated rats and esophagitis rats enabled us to identify differentially regulated genes associated with esophagitisinduced inflammation. Among the 1,031 probes on this array, there were 368 probes $(36 \%)$ that showed a $>1.5$-fold difference in expression between the sham-operated and esophagitis groups 2 weeks after the GDE operation; 185 probes (17.9\%) were up-regulated and 183 probes $(17.7 \%)$ were downregulated. Tables I and II list the genes known to be up- or down-regulated $\geq 1.5$-fold after the operation as compared to 
Table III. Caronical pathways significantly affected in the esophageal mucosa.

\begin{tabular}{lcll}
\hline & & \multicolumn{2}{c}{ Genes } \\
\cline { 3 - 4 } Pathway & Significance & \multicolumn{1}{c}{ Up-regulated } & \multicolumn{1}{c}{ Down-regulated } \\
\hline IL-6 signaling & $2.18 \times 10^{-6}$ & COL1A1, I11A, JUN, MAP2K2, MAPK14, & CYP19A1, FOS, HSPB1, MAPK9, \\
& & NFKB1, STAT3, TNF & NRAS, TNFRSF1B \\
p38 MAPK signaling & $7.57 \times 10^{-3}$ & IL1A, MAPK14, TGFB3, TNF & HSPB1, MAPK12, MYC, TNFRSF1B \\
IL-2 signaling & $1.06 \times 10^{-2}$ & IL2, IL2RB, JUN, MAP2K2 & FOS, NRAS \\
\hline
\end{tabular}

COL1A1: collagen, type I, a 1; CYP19A1: cytochrome P450, family 19, subfamily A, polypeptide 1; FOS: v-fos FBJ murine osteosarcoma viral oncogene homolog; HSPB1: heat shock $27 \mathrm{kDa}$ protein 1; IL1A: interleukin 1, $\alpha$; IL2: interleukin 2; IL2RB: interleukin 2 receptor, B; JUN: v-jun sarcoma virus 17 oncogene homolog (avian); MAP2K2: mitogen-activated protein kinase kinase 2; MAPK9: mitogen-activated protein kinase 9; MAPK12: mitogen-activated protein kinase 12; MAPK14: mitogen-activated protein kinase 14; MYC: v-myc myelocytomatosis viral oncogene homolog (avian); NFKB1: nuclear factor of $\kappa$ light polypeptide gene enhancer in B-cells 1 (p105); NRAS: neuroblastoma RAS viral (v-ras) oncogene homolog; STAT3: signal transducer and activator of transcription 3; TGFB3: transforming growth factor, $\beta 3$; TNF: tumor necrosis factor (TNF superfamily, member 2); TNFRSF1B: tumor necrosis factor receptor superfamily, member $1 \mathrm{~B}$.

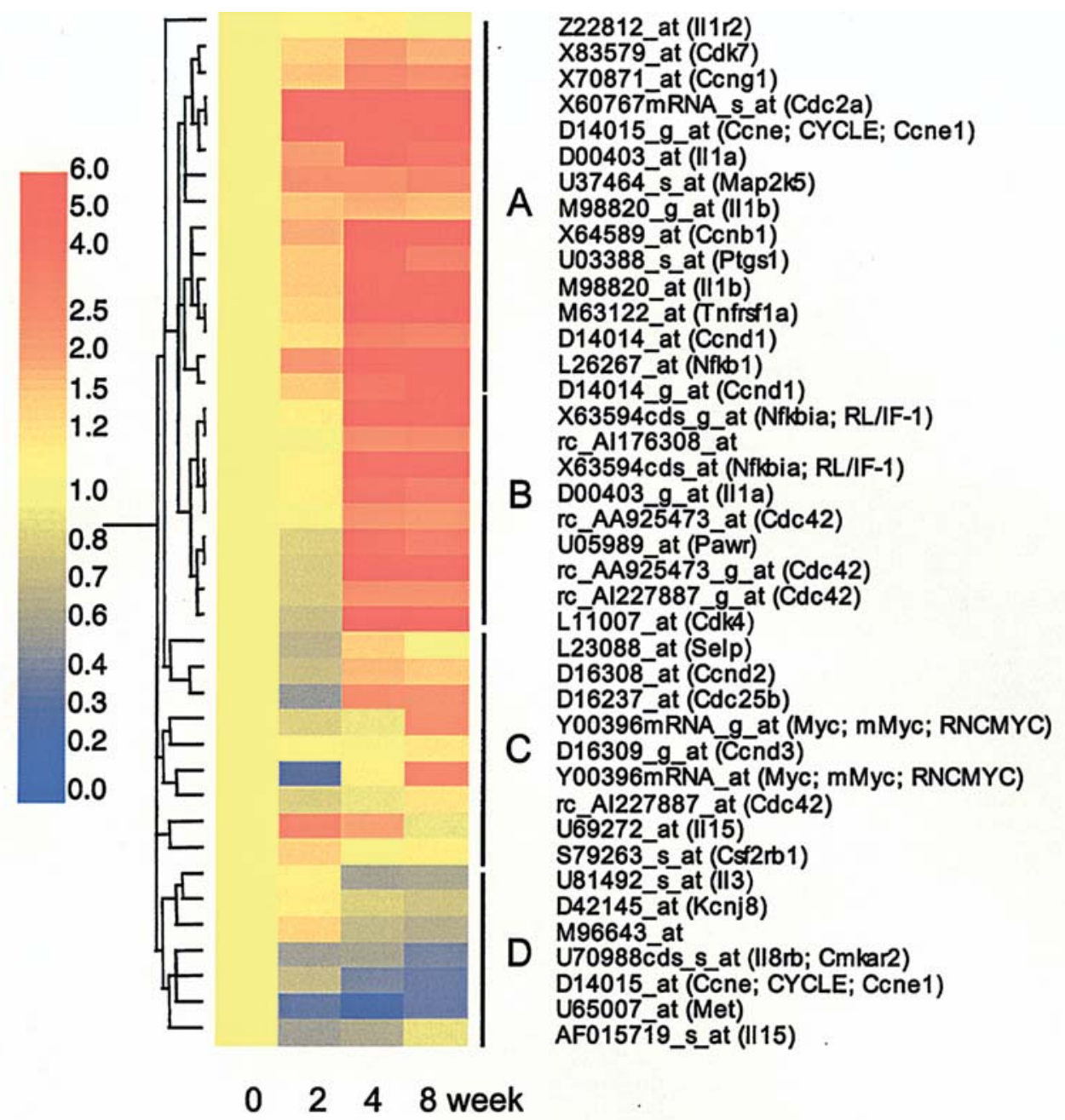

Figure 1. Expression clusters of cytokine-related genes in esophageal mucosa after esophagogastroduodenostomy in rats. In the heirarchical-clustering analysis, a fold-change ratio was calculated using the sham-operated rats and esophagitis samples. Red indicates up-regulated genes, and blue indicates downregulated genes. Yellow indicates the same expression level as the normoxia sample.

the sham-operated group. Genes that were determined as 'no change' according to Affymetrix algorithms were excluded from Tables I and II. Genes that were up-regulated $\geq 1$.5-fold were involved in cellular stress responses [heat shock protein 60, 70, and Dna-J, apoptosis-related genes (caspase 2, 3, 6, bcl-2 asscociated death agonist), transcriptional factors (nuclear 


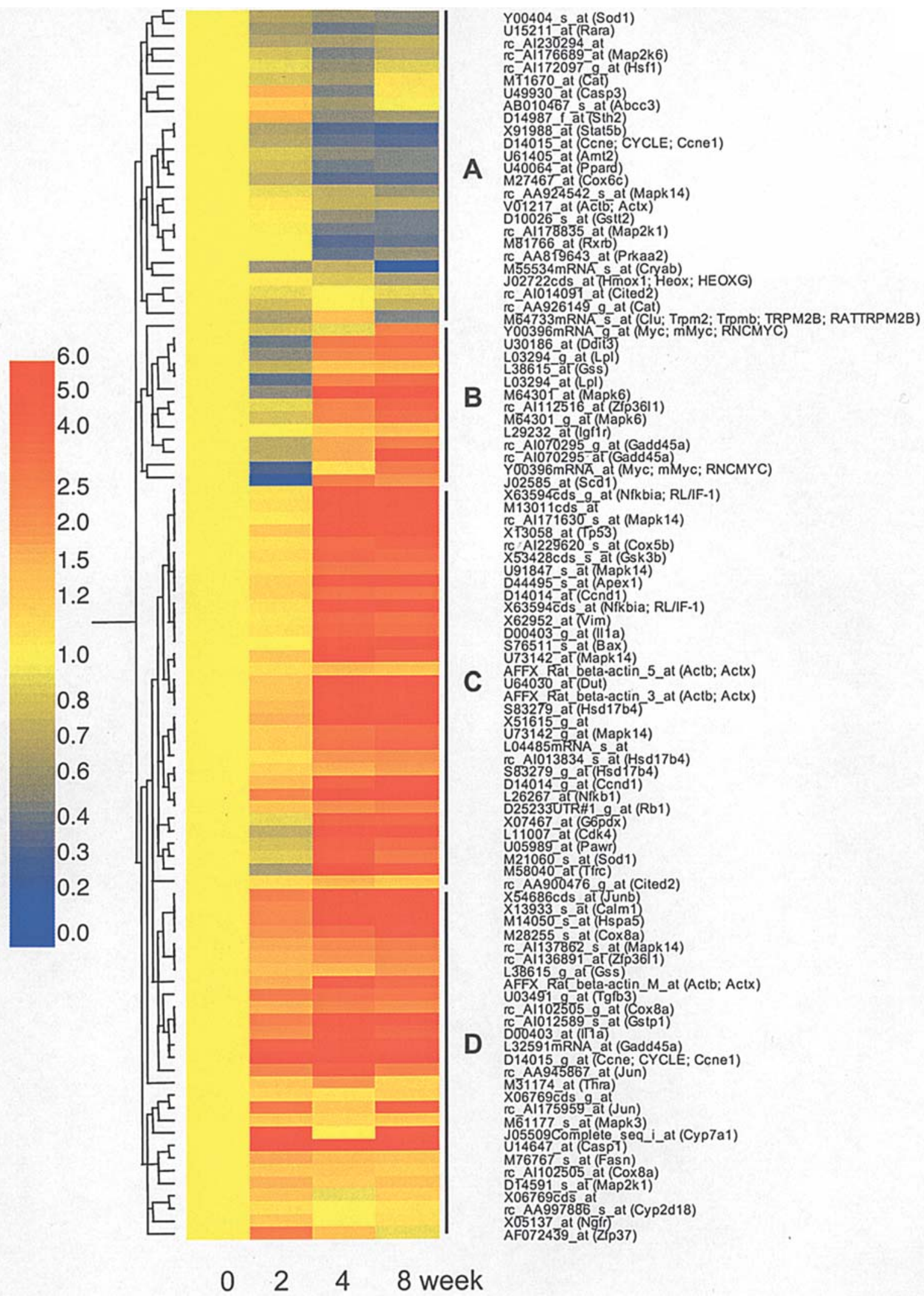

Figure 2. Expression clusters of transcriptional factor-related genes in esophageal mucosa after esophagogastroduodenostomy in rats. In the hierarchicalclustering analysis, a fold-change ratio was calculated using the sham-operated rats and esophagitis samples. Red indicates up-regulated genes, and blue indicates down-regulated genes. Yellow indicates the same expression level as the normoxia sample.

factor $\kappa \mathrm{B})$, DNA damage-associated genes (mismatch repair protein), and enzymes (cytochrome c oxidase, glutathione stransferase)].
To further refine the list of esophagitis-affected genes, we next investigated which of these genes are known to interact biologically. To this end we carried out pathway analysis on the 
above 368 up- and down-regulated genes using an Ingenuity pathway analysis (IPA) tool. Of these genes, 207 mapped to genetic networks, as defined by the IPA tool. Three caronical networks associated with inflammation were found to be significant in that they had more of the identified genes present than would be expected by chance (Table III): IL-6 signaling, p38 MAPK signaling, and IL-2 signaling. We recently found that the expression of IL-6 mRNA is increased in patients with reflux esophagitis, especially in endoscopic negative reflux disease (unpublished data). In addition, the role of IL-6 in esophagitis was reported by Cheng et al (8), who demonstrated that acid-induced platelet activating factor (PAF) formation induces the production of IL-6 in esophageal mucosa. As IL-6 signaling molecules were most significantly affected in this model of esophagitis, further studies will be necessary to clarify the role of this signaling in the pathogenesis of GERD. In addition, our group recently investigated the role of p38 MAPK in the production of IL-8 by human esophageal epithelial cells $(4,9)$. We found that trypsin or bile acids induce p38 MAPK phosphorylation, determined by Western blotting, and that FR167653, a specific inhibitor of p38 MAPK, significantly inhibits IL-8 production elicited by these stimuli, indicating that trypsin or bile acids induce IL- 8 production from esophageal cells via p38 MAPK pathways, which may be involved in the pathogenesis of reflux esophagitis. Further studies will be needed to clarify the role of IL-6, p38 MAPK or IL-2, and especially intracellular signaling, in the combinedtype of esophagitis.

In order to focus on inflammation, the expression of genes to be studied was narrowed down to 40 and 102 probes, which were selected using the following keywords: cytokine and transcriptional factor, respectively, using a soft of NetAffx analysis center (Affymetrix). In this study, the expression of these probes at each time point after the GDE operation was shown to be within the limits of 6.0-fold up-regulation (red) or down-regulation (blue) compared to that in the shamoperated group (yellow) using a GeneSpring software. Fig. 1 shows that the probes associated with 'cytokine' were divided into several clusters on the basis of time-kinetics. In group A (Fig. 1), many genes, including the early-response genes (IL-1 and cyclooxygenase), were up-regulated predominantly during the experiment for 8 weeks. In particular, the expression of many probe sets for interleukin (IL-1 $\alpha$, IL-1ß, IL-1 receptor) was markedly up-regulated, and their expression continued or was even further enhanced during the period of esophagitis. In group $\mathrm{B}$, nuclear factor- $\mathrm{K} \mathrm{B}$ and cell cycle-related genes [cell division cycle 42 homolog $(\mathrm{Cdc} 42)$ and cyclin-dependent kinase $4(\mathrm{Cdk} 4)$ ] were up-regulated at 4 and 8 weeks after the operation. These increases in cell cycle-related genes may result in hyperplasia of the epidermis and basal cells of the esophagus, which are features of esophagitis described in previous reports $(5,10,11)$. The gene expression of group $\mathrm{C}$ showed irregular changes during the experiment. Group D included genes such as IL-3 and Met that were down-regulated at least two points compared to the sham.

Fig. 2 shows that the probes associated with 'transcriptional factor' were also divided into several clusters on the basis of time-kinetics. In group A (Fig. 2), many genes were downregulated at least two points compared to the sham, including CuZn-superoxide dismutase (SOD1), catalase (Cat), and heme oxygenase-1 (Hmox-1). The down-regulation of these anti-oxidative genes may be associated with aggravation of esophageal injury. Oh et al (10) have demonstrated the increase of malondialdehyde (MDA), an index of lipid peroxidation, and the mucosal depletion of reduced glutathione in the esophagus of reflux esophagitis. Further studies are needed to clarify the role of oxygen radicals and antioxidants in reflux esophagitis. The gene expression of group B was decreased at 2 weeks after the operation and then increased in the late period of the experiment, as did growth arrest and DNA-damage-inducible $45 \alpha(\operatorname{Gadd} 45 \alpha)$ and mitogen-activated protein kinase 6 (Mapk6). Gadd45 $\alpha$ is a member of a group of genes induced by agents that damage DNA and/or cause growth arrest. This study showed that three probes of GADD45 $\alpha$ (rc_AI070295_at, rc_AI070295_g_at, L32591mRNA_at) in this array were upregulated after the operation in comparison with the shamoperated rats. Increased GADD45a gene expression has been detected in many mammalian cell types and has been implicated in terminal differentiation (12), growth suppression (13), and apoptosis (14). Previous studies suggested that GADD45 may mediate genotoxic stress-induced apoptosis via activation of c-Jun N-terminal kinase (JNK) and/or p38 MAPK $(15,16)$. These data, including the present information, suggest that enhanced expression of GADD45 followed by MAPK activation may be involved in the apoptosis of esophageal epithelial cells after the operation. In groups $\mathrm{C}$ and $\mathrm{D}$, many genes, including early- and late-response genes, were up-regulated. Group C (late-response genes) included NF-кB and Mapk14, and group D included v-jun (Jun), heat shock $70 \mathrm{kD}$ protein 5 (Hspa5), glutathione-S-transferase, pi 1 (Gstp1), and several forms of MAPK. This up-regulation of inflammation-associated transcriptional factor may support the hypothesis that esophageal epithelial cells respond by immune and inflammatory pathways after exposure to gastroduodenal refluxates. In particular, NF- $\mathrm{NB}$ activation leads to expression of a large number of NF- $\mathrm{kB}$-dependent genes, which are important mediators of immune and inflammatory responses, including tumor necrosis factor (TNF)- $\alpha$, IL-1, IL-6, IL-8, IL-10, granulocyte-macrophage colony-stimulating factor, inducible nitric oxide synthase, intercellular adhesion molecule 1 (ICAM-1), E-selectin, vascular cell adhesion molecule 1 (VCAM-1), and MHC class I and II molecules. The activation of $\mathrm{NF}-\kappa \mathrm{B}$ has been demonstrated in experimental esophagitis (10), endoscopic negative reflux disease (17), and in Barrett's epithelium and adenocarcinoma (18). These results, including the present data, suggest that the inhibition or regulation of the $\mathrm{NF}-\kappa \mathrm{B} /$ proinflammatory cytokine pathway may be an important target for future therapeutic strategies. Detailed clinical studies of the interaction between esophageal epithelium and gastric/duodenal refluxate should make it possible to identify a key therapeutic target molecule that regulates esophageal inflammation.

\section{References}

1. Fitzgerald RC, Onwuegbusi BA, Bajaj-Elliott M, Saeed IT, Burnham WR and Farthing MJ: Diversity in the oesophageal phenotypic response to gastro-oesophageal reflux: immunological determinants. Gut 50: 451-459, 2002.

2. Isomoto H, Wang A, Mizuta Y, et al: Elevated levels of chemokines in esophageal mucosa of patients with reflux esophagitis. Am J Gastroenterol 98: 551-556, 2003. 
3. Yoshida N, Uchiyama K, Kuroda M, et al: Interleukin-8 expression in the esophageal mucosa of patients with gastroesophageal reflux disease. Scand J Gastroenterol 39: 816-822, 2004.

4. Imamoto E, Yoshida N, Uchiyama K, et al: Effects of bile acids and acidic exposure on IL-8 expression in human esophageal epithelial cells. Gastroenterology 124: A-409, 2003.

5. Naito Y, Uchiyama K, Kuroda M, et al: Role of pancreatic trypsin in chronic esophagitis induced by gastroduodenal reflux in rats. J Gastroenterol 41: 198-208, 2006.

6. Naito Y, Uchiyama K, Kuroda M, et al: Laser capture microdissection/GeneChip analysis of gene expression in glomerular cells in diabetic db/db mice. Redox Rep 9: 307-312, 2004.

7. Naito Y, Mizushima K and Yoshikawa T: Global analysis of gene expression in gastric ischemia-reperfusion: a future therapeutic direction for mucosal protective drugs. Dig Dis Sci 50 (suppl 1): S45-S55, 2005.

8. Cheng L, Cao W, Fiocchi C, et al: HCl-induced inflammatory mediators in cat esophageal mucosa and inflammatory mediators in esophageal circular muscle in an in vitro model of esophagitis increased release of interleukin- 6 by oesophageal mucosa in children with reflux oesophagitis. Am J Physiol Gastrointest Liver Physiol 11: 839-843, 2006.

9. Yoshida N, Katada K, Kuroda M, et al: Trypsin activates human esophageal epithelial cells to produce inflammatory cytokines through protease-activated receptor 2. Gastroenterology 126: A-501, 2004.

10. Oh TY, Lee JS, Ahn BO, et al: Oxidative damages are critical in pathogenesis of reflux esophagitis: implication of antioxidants in its treatment. Free Radic Biol Med 30: 905-915, 2001.
11. Zhang F, Altorki NK, Wu YC, Soslow RA, Subbaramaiah K and Dannenberg AJ: Duodenal reflux induces cyclooxygenase-2 in the esophageal mucosa of rats: evidence for involvement of bile acids. Gastroenterology 121: 1391-1399, 2001

12. Liebermann DA and Hoffman B: Myeloid differentiation (MyD) primary response genes in hematopoiesis. Blood Cells Mol Dis 31: 213-228, 2003.

13. Jin S, Antinore MJ, Lung FD, et al: The GADD45 inhibition of $\mathrm{Cdc} 2$ kinase correlates with GADD45-mediated growth suppression. J Biol Chem 275: 16602-16608, 2000.

14. Sheikh MS, Hollander MC and Fornance AJ Jr: Role of Gadd45 in apoptosis. Biochem Pharmacol 59: 43-45, 2000.

15. Takekawa M and Saito H: A family of stress-inducible GADD45like proteins mediate activation of the stress-responsive MTK1/ MEKK4 MAPKKK. Cell 95: 521-530, 1998.

16. Mita H, Tsutsui J, Takekawa M, Witten EA and Saito H: Regulation of MTK1/MEKK4 kinase activity by its N-terminal autoinhibitory domain and GADD45 binding. Mol Cell Biol 22: 4544-4555, 2002.

17. Isomoto H, Nishi $\mathrm{Y}$, Wang A, et al: Mucosal concentrations of proinflammatory cytokines and chemokines at gastric cardia: implication of Helicobacter pylori infection and gastroesophageal reflux. Am J Gastroenterol 99: 1063-1068, 2004

18. O'Riordan JM, Abdel-latif MM, Ravi N, et al: Proinflammatory cytokine and nuclear factor kappa-B expression along the inflammation-metaplasia-dysplasia-adenocarcinoma sequence in the esophagus. Am J Gastroenterol 100: 1257-1264, 2005. 